\title{
ALMOST PERIODIC SOLUTIONS FOR A CERTAIN CLASS OF ALMOST PERIODIC SYSTEMS
}

\author{
GEORGE SEIFERT
}

\begin{abstract}
Using a result due to Medvedev [3], we obtain conditions under which systems of ordinary differential equations of the form $x^{\prime}=F(t, x, x)+G(t, x)$ where $F$ and $G$ are almost periodic in $t$ will have unique almost periodic solutions with certain global stability properties and module containment. These conditions are compared to conditions for the existence, but not uniqueness, for such solutions obtained by Kartsatos in [2]. Both results, our as well as Kartsatos', are applied to a second order equation of Lienard type with almost periodic forcing.
\end{abstract}

In [2], Kartsatos uses a result due to Medvedev [3] to get conditions sufficient for the existence of an almost periodic solution of a differential equation of the form

$$
x^{\prime}=F(t, x, x)+G(t, x)
$$

here $x, F$, and $G$ are real $n$-vectors, the set of which we denote by $R^{n}$, and $F$ and $G$ are almost periodic (a.p. for short) in $t$ uniformly for $x$ in compact subsets of $R^{n}$. It must also be assumed that $F(t, x, 0) \equiv 0$, a condition not mentioned in [2]. This is no great restriction, since if it does not hold, we consider (1) with $F(t, x, x)$ replaced by $F(t, x, x)-F(t, x, 0)$ and $G(t, x)$ by $G(t, x)+F(t, x, 0)$. However, the above-mentioned result in [2] involves conditions on $G$ which of course may change if $F(t, x, 0) \neq 0$. For a more explicit discussion of Kartsatos' result, cf. Remark 3 of this paper.

Uniqueness of the a.p. solution, however, is not implied in Kartsatos' result. This is interesting since many of the known sufficient conditions for the existence of a.p. solutions involve some kind of uniform asymptotic stability for a solution bounded on $R$, the set of reals, which in turn usually implies the uniqueness of the a.p. solution with respect to some bounded set in $R^{n}$ containing its values; cf. for example $[1,4]$ for more details on such results. Clearly, some sort of uniqueness and stability for an a.p. solution would be desirable in practical applications. Accordingly, it is the purpose of this paper to establish a condition for the existence of a unique a.p. solution with certain stability properties.

Let $f(t, x)$ be continuous on $R \times R^{n}$ to $R^{n}$. The following is a nonglobal version of Medvedev's result in [3] and is proved by making some obvious modifications in the proof in [3]. In what follows, $|x|$ will denote any convenient norm in $R^{n}$.

Received by the editors January 27, 1981.

1980 Mathematics Subject Classification. Primary 34C27.

Key words and phrases. Almost periodic systems, almost periodic solutions.

(c) 1982 American Mathematical Society 0002-9939/82/0000-0011/\$02.25 
THEOREM 1. Let $|f(t, 0)| \leqslant M$ for $t \in R$ and let there exist $H>0, p>0$ with $p H<1$, and $r>M / p$ such that

$$
|x-y+h(f(t, x)-f(t, y))| \leqslant(1-p h)|x-y|
$$

holds for $0<h<H,|x| \leqslant r,|y| \leqslant r$, and $t \in R$. Then there exists a unique solution $\bar{x}(t)$ of

$$
x^{\prime}=f(t, x)
$$

such that $|\bar{x}(t)| \leqslant M / p$ for $t \in R$. Also if $x(t)$ is any solution of (2) such that, for some $t_{0},\left|x\left(t_{0}\right)\right|<M / p$, then $x(t)-\bar{x}(t) \rightarrow 0$ as $t \rightarrow \infty$.

The proof involves writing (2) in the form $x^{\prime}=A(t, x)+b(t)$ where $A(t, x)=$ $f(t, x)-f(t, 0)$ and $b(t)=f(t, 0)$ and following the proof of the corresponding result in [3]; we omit the details.

The next theorem uses an existence result due basically to Amerio; cf. either [1] or [4] for details.

THEOREM 2. Let $f$ satisfy the conditions in Theorem 1 and also be a.p. in $t$ uniformly for $x$ in compact subsets of $R^{n}$ (cf. [1] or [4] for a definition). Then there exists a unique a.p. $\bar{x}(t)$ of (2) such that $|\bar{x}(t)| \leqslant M / p$ for $t \in R$ and such that if $x\left(t_{0}\right)<M / p$ for some $t_{0}$ and solution $x(t)$ of (2), then $x(t)-\bar{x}(t) \rightarrow 0$ as $t \rightarrow \infty$. Also the frequency module of $\bar{x}(t)$ is contained in the frequency module of $f(c f .[1]$ or [4] for a definition).

We again omit the details of the proof, except to point out that if $g(t, x)$ is in the hull of $f(t, x)$; i.e., $g(t, x)=\lim f\left(t+t_{k}, x\right)$ for some sequence $\left\{t_{k}: k=1,2, \ldots\right\}$, the limit being uniform on sets $R \times K, K \subset R^{n}, K$ compact, then $g$ satisfies the same conditions imposed on $f$ in Theorem 2.

Our main result is for equations of the form (1). We assume henceforth that there exist positive numbers $r, H, p$ with $p H<1$, and $L_{1} \geqslant 0, L_{2}>0, M_{1}>0$ such that

(i) $|x-y+h(F(t, u, x)-F(t, u, y))| \leqslant(1-p h)|x-y|$ for $0<h<H,|x|<$ $r,|y|<r,|u| \leqslant r, t \in R$.

(ii) $|G(t, x)-G(t, y)| \leqslant L_{1}|x-y|$ for $|x| \leqslant r,|y| \leqslant r, t \in R$.

(iii) $|F(t, u, x)-F(t, v, x)| \leqslant L_{2}|u-v|$ for $|x| \leqslant r,|u| \leqslant r,|v|<r, t \in R$.

(iv) $|F(t, 0,0)+G(t, 0)| \leqslant M_{1}$ for $t \in R$.

Lemma 1. Let (i)-(iii) hold and suppose $p>L_{1}+L_{2}$. Then there exist positive numbers $p_{1}$ and $H_{1}, p_{1} H_{1}<1$, such that

$$
|x-y+h(f(t, x)-f(t, y))|<\left(1-p_{1} h\right)|x-y|
$$

for $|x|<r,|y| \leqslant r, 0<h<H_{1}$, and $t \in R$; here $f(t, x)=F(t, x, x)+G(t, x)$.

Proof. For $|x| \leqslant r,|y| \leqslant r, t \in R$, we have

$$
\begin{aligned}
\mid x-y+h & (F(t, x, x)+G(t, x)-F(t, y, y)-G(t, y)) \mid \\
& \leqslant|x-y+h(F(t, x, x)-F(t, y, y))|+h L_{1}|x-y| \\
& \leqslant|x-y+h(F(t, x, x)-F(t, x, y))|+h\left(L_{2}+L_{1}\right)|x-y| \\
& \leqslant\left(1-h\left(p-L_{1}-L_{2}\right)\right)|x-y| .
\end{aligned}
$$


We now take $p_{1}=p-L_{1}-L_{2}$, and $H_{1}>0$ such that $p_{1} H_{1}<1$ and the proof is complete.

THEOREM 3. Let (i)-(iv) hold and suppose $p_{1}=p-L_{1}-L_{2}>M_{1} / r$. Let $F(t, x, u)$ and $G(t, x)$ be a.p. in $t$ uniformly for $x$ and $u$ in compact subsets of $R^{n}$. Then there exists a unique a.p. solution $\bar{x}(t)$ of (1) such that $|\bar{x}(t)| \leqslant M_{1} / p_{1}$ for $t \in R$ whose frequency module is contained in the frequency module of $F(t, x, u)+G(t, x)$, and if $x(t)$ is any solution of (1) with $\left|x\left(t_{0}\right)\right| \leqslant M_{1} / p_{1}$ for some $t_{0}$, then $x(t)-\bar{x}(t) \rightarrow$ 0 as $t \rightarrow \infty$.

Proof. Under the hypotheses above, the hypotheses of Theorem 2 hold with $f$ as defined in Lemma $1, M=M_{1}, p=p_{1}$, and $H=H_{1}$; our theorem thus is a direct consequence of Theorem 2.

REMARK 1. Note that $L_{1}$ and $L_{2}$ depend in general on $r$; in fact, the choice of $p$ in (i) may also depend on $r$. Thus the condition $p-L_{1}-L_{2}>M_{1} / r$ can be regarded as a condition on $r$. In the following example this will become clear.

REMARK 2. The unique a.p. solution of Theorem 2 and hence also Theorem 3 actually is uniformly asymptotically stable in the sense of Liapunov for any solution $y(t)$ such that $\left|y\left(t_{0}\right)\right|<r$ for some $t_{0}$; this follows from the proof of Theorem 1.

REMARK 3. The main result (Theorem 2) in [2] shows that in addition to condition (i), the following conditions are sufficient for the existence of an a.p. solution of (1), but not necessarily its uniqueness. Define

$$
\begin{aligned}
& B(t, u)=G(t, u)+F(t, u, 0), \\
& M(r)=\sup \{|B(t, u)|:|u| \leqslant r, t \in R\}, \text { and } \\
& L(r)=\inf \{L:|B(t, u)-B(t, v)| \leqslant L|u-v|,|u| \leqslant r,|v| \leqslant r, t \in R\} .
\end{aligned}
$$

Then

(ii') $L(r)<p$ and

(iii') $M(r) / r<p$

where $p$ and $r$ are as in (i).

It is not clear that the hypotheses of our theorem imply those of Theorem 2 in [2]. This will be seen in the following example.

We consider a system in $R^{2}$ of the form

$$
x_{1}^{\prime}=a x_{2}-x_{1}, \quad x_{2}^{\prime}=-x_{2}-g\left(x_{1}\right)+b(t)
$$

where $a$ is a constant, $0<a<1, g(0)=0, g$ is locally Lipschitz on $R$, and $b(t)$ is a.p.

It is not difficult to show that (4) is equivalent to the second order scalar equation

$$
x^{\prime \prime}+2 x^{\prime}+x+a g(x)=a b(t) .
$$

For each $r>0$ we define $L_{g}(r)$ to be the infimum of the set of $L$ such that

$$
|g(x)-g(y)| \leqslant L|x-y| \text { for }|x| \leqslant r,|y| \leqslant r .
$$

Clearly (6) holds for $L=L_{g}(r)$. 
THEOREM 4. Let $M_{1}=\sup \{|b(t)|: t \in R\}$ and suppose there exists $r>0$ such that

$$
M_{1} / r+\max \left\{a, L_{g}(r)\right\}<1 .
$$

Then there exists a unique a.p. solution $\left(\bar{x}_{1}(t), \bar{x}_{2}(t)\right)$ of $(4)$ such that $\left|\bar{x}_{1}(t)\right|+\left|\bar{x}_{2}(t)\right|$ $<M_{1} / p_{1}$ for $t \in R$ where $p_{1}=1-\max \left\{a, L_{g}(r)\right\}$ and each solution $\left(x_{1}(t), x_{2}(t)\right)$ such that $\left|x_{1}\left(t_{0}\right)\right|+\left|x_{2}\left(t_{0}\right)\right| \leqslant M_{1} / p_{1}$ for some $t_{0}$, satisfies $x_{1}(t) \rightarrow \bar{x}_{1}(t)$ and $x_{2}(t) \rightarrow$ $\bar{x}_{2}(t)$ as $t \rightarrow \infty$.

Proof. Define

$$
F(t, u, x)=\left(a u_{2}-x_{1},-x_{2}-g\left(u_{1}\right)\right), \quad G(t, x)=(0, b(t))
$$

where $x=\left(x_{1}, x_{2}\right), u=\left(u_{1}, u_{2}\right)$, and define the norm $|x|=\left|x_{1}\right|+\left|x_{2}\right|$ for any $x \in R^{2}$.

If we take $p=1,0<H<1$, it is easy to see that (i) holds. Clearly if we take $L_{1}=0$ and $L_{2}=\max \left\{a, L_{g}(r)\right\}$, then (ii) and (iii) also hold. Finally (iv) holds from the definition of $M_{1}$ given in our theorem.

It remains to check that $p-L_{1}-L_{2}>M_{1} / r$, but since $p=1$, this is a trivial consequence of (7). Hence our theorem follows as a direct consequence of Theorem 3.

COROllary 1. Suppose $g(x)$ is continuously differentiable in a neighborhood of $x=0$ and $g^{\prime}(0)=0$. Then for $M_{1}$ sufficiently small, (4) has a unique a.p. solution with values in some set in $R^{2}$ of the form $|x|<r_{0}, r_{0}>0$, and which is approached by solutions with values in that set as $t \rightarrow \infty$.

Proof. Since now $L_{g}(r) \rightarrow 0$ as $r \rightarrow 0$, and $0<a<1$, we may fix $r=r_{1}$ such that $L_{g}\left(r_{1}\right)<a$, and then for $M_{1}$ sufficiently small, (7) will hold. Q.E.D.

Another very simple corollary of Theorem 4 would be obtained if $L_{g}(r)<m<1$ for $r>0$; in this case for any $M_{1}$, i.e., any a.p. function $b(t)$, (4) has a unique a.p. solution $\bar{x}(t)$ such that $|\bar{x}(t)| \leqslant M_{1} /\left(1-m_{0}\right)$ for all $t \in R$ where $m_{0}=\max \{a, m\}$. Note that this condition implies that $|g(x)| \leqslant m$ for all $x$.

Let us now derive conditions for the existence of an a.p. solution of (4) using Theorem 2 in [2]; cf. Remark 3.

Define $F(t, u, x)$ and $G(t, x)$ as in the proof of Theorem 4. Then clearly (i) holds for arbitrary $r>0$ if $p=1$ and $0<H<1$. Also we find

$$
B(t, u)=\left(a u_{2},-g\left(u_{1}\right)+b(t)\right),
$$

where $u=\left(u_{1}, u_{2}\right)$. Thus

$$
M(r)=\sup \left\{a\left|u_{2}\right|+\left|g\left(u_{1}\right)-b(t)\right|:\left|u_{1}\right|+\left|u_{2}\right|<r, t \in R\right\}
$$

and

$$
\begin{array}{r}
L(r)=\inf \left\{L: a\left|u_{2}-v_{2}\right|+\left|g\left(u_{1}\right)-g\left(v_{1}\right)\right|<L\left(\left|u_{1}-v_{1}\right|+\left|u_{2}-v_{2}\right|\right),\right. \\
\left.\left|u_{1}\right|+\left|u_{2}\right|<r,\left|v_{1}\right|+\left|v_{2}\right|<r\right\} .
\end{array}
$$

If $g_{r}=\sup \{|g(u)|:|u| \leqslant r\}$, and $M_{1}$ is as above, then $M(r)<a r+g_{r}+M_{1}$ and since $g_{r}<L_{g}(r) r$, where $L_{g}(r)$ is as previously defined, and $p=1$, it follows that (iii') in Remark 3 holds if

$$
a+L_{g}(r)+M_{1} / r<1
$$


Also clearly $L(r)<\max \left\{a, L_{g}(r)\right\}$, and since $0<a<1$, (ii') in Remark 3 holds if $L_{g}(r)<1$, which is implied by (10). So if (10) holds for some $r$, Theorem 2 of [2] implies the existence of an a.p. solution of (4).

It can easily be seen that (10) is best possible to obtain (ii') and (iii') for general $g$ and $b$ such as occur in (4). However, (10) is not implied by (7) of our Theorem 4; suppose $M_{1} / r=\frac{1}{4}, a=L_{g}(r)=\frac{1}{2}$. It therefore follows that Theorem 4 can yield the stronger uniqueness and stability property of the a.p. solution in cases where Theorem 2 in [2] does not. For other results on systems like (4), cf. [1].

Some of the following observations were suggested by the referee.

First, it can easily be shown that (1.1) implies

$$
|f(t, x)-f(t, y)|>p|x-y|
$$

for $|x|<r,|y|<r, t \in R$. A similar condition on $F$ is implied by (i). This clearly imposes some restrictions on the equations we consider.

Second, conditions (1.1) and (i) depend on the choice of norm in $R^{n}$. If we use the Euclidean norm with inner product denoted by $\langle x, y\rangle$, it follows easily from (1.1) that

$$
\langle x-y, f(t, x)-f(t, y)\rangle\left\langle-p|x-y|^{2}\right.
$$

for $|x|<r,|y| \leqslant r, t \in R$. So if $x(t)$ and $y(t)$ are solutions of (2) such that $|x(t)|<r,|y(t)|<r$ for $t \geqslant t_{0}$, then it follows from (12) that

$$
|x(t)-y(t)| \leqslant\left|x\left(t_{0}\right)-y\left(t_{0}\right)\right| \exp \left(-p\left(t-t_{0}\right)\right)
$$

for $t \geqslant t_{0}$. Thus the convergence $x(t)-\bar{x}(t) \rightarrow 0$ as $t \rightarrow \infty$ is actually uniform (in $t_{0}$ ) and exponential. This can also be seen by considering a proof of Theorem 1 in terms of a general norm in $R^{n}$, but not in as simple a fashion.

A final remark: while Theorem 2 in [2] does not assert the existence of a unique a.p. solution of (1), it does assert frequency module containment. Also the proof of this theorem does not appeal to Amerio separation arguments or uniform stability conditions directly but uses Medvedev's condition (i), in a sense a stability condition, and the Bohr definition of a.p. functions in term of translation numbers; again cf. [1, pp. 60-63] for details on frequency modules for a.p. functions.

\section{REFERENCES}

1. A. M. Fink, Almost periodic differential equations, Lecture Notes in Math., vol. 377, Springer-Verlag, Berlin and New York, 1974.

2. A. G. Kartsatos, Almost periodic solutions to nonlinear systems, Boll. Un. Mat. Ital. 9 (4) (1974), 10-15.

3. N. V. Medvedev, Certain tests for the existence of bounded solutions of systems of differential equations, Differencial'nye Uravneniya 4 (1968), 1258-1264.

4. T. Yoshizawa, Stability theory and the existence of periodic solutions and almost periodic solutions, Lectures in Appl. Math., vol. 14, Springer-Verlag, Berlin and New York, 1975.

Department of Mathematics, Iowa State University, Ames, Iowa 50011 